\title{
Refractive index sensing measurement based on periodically tapered small core singlemode fibre
}

\author{
Pengfei Wang ${ }^{1,2^{*}}$ Gilberto Brambilla ${ }^{1}$, Ming Ding ${ }^{1}$, Lin Bo $^{2}$, Yuliya Semenova ${ }^{2}$, Qiang Wu ${ }^{2}$ and \\ Gerald Farrell $^{2}$ \\ ${ }^{1}$ Optoelectronics Research Centre, University of Southampton, Southampton SO17 1BJ, United \\ Kingdom \\ ${ }^{2}$ Photonics Research Center, School of Electronic and Communications Engineering, Dublin \\ Institute of Technology, Kevin Street, Dublin 8, Dublin Ireland
}

\begin{abstract}
An all-fibre refractive index sensor with a simple periodical tapers configuration is proposed and investigated experimentally. The proposed fibre refractive index sensor consists of a small core singlemode fibre with tapers periodically fabricated along the fibre using a focused $\mathrm{CO}_{2}$ laser beam, and sandwiched between two standard singlemode fibres. Such a structure can be used for sensing of refractive index by measuring the dip wavelength shift of the multimode interference within the small core fibre cladding. A minimum sensitivity of $125 \mathrm{~nm} / \mathrm{RIU}$ is measured for a refractive index of 1.33 and a maximum sensitivity of $383 \mathrm{~nm} / \mathrm{RIU}$ for a refractive index of 1.38 . The proposed refractive index sensor benefits from simplicity and low-cost and achieves a competitive sensitivity compared with other fibre-optic sensors.
\end{abstract}

Keywords: Fibre optic sensor, refractive index sensor, mode interference

\section{INTRODUCTION}

Fibre refractive index (RI) sensors offer advantages such as immunity to electromagnetic interference, high sensitivity, fast response, small size and ease of fabrication. To date several types of optical fibre refractometers have been proposed for applications in growth areas for RI sensing ${ }^{1-4}$. The most common approaches are a fibre Bragg grating ${ }^{1}$, long period grating $^{2}$, tapered microfibre ${ }^{3}$ and singlemode fibre (SMF)-multimode fibre (MMF)-singlemode ${ }^{4}$ based fibre refractometers. As an alternative to these existing fibre refractometers, an SMF28 -small core -SMF28 (SSCS) fibre structure based fibre refractometer ${ }^{5}$ has been proposed recently featuring a high sensitivity of $102 \mathrm{~nm} / \mathrm{RIU}$ at an $\mathrm{RI}=1.324$.

For RI sensing, an SSCS structure proposed in Ref. 5 needs strong multimode interference between the fibre cladding modes, thus the diameter of the cladding of the small core fibre in an SSCS structure should be small in order to achieve a higher sensitivity. Experimentally, an effective approach is to employ a small core fibre with a smaller cladding diameter achieved by etching using hydrofluoric acid, but this increases the fabrication difficulty and the induced surface roughness may lead to a significant discrepancy between experimental results and theoretical design ${ }^{4}$. Alternatively, fibre tapering can replace the chemical etching process; since light guided in a tapered fibre has a

"pw3y09@orc.soton.ac.uk; phone: +44 23 8059 3954; fax: +44 2380593149 
significant fraction of power propagating as an evanescent wave, and therefore the effective index of the guided mode is affected by the surrounding medium RI. It is well known that the fraction of power in the form of evanescent field, and thus its sensitivity to environmental changes, increases for small taper diameters.

In this paper, we report the experimental demonstration of a novel high sensitivity refractometric sensor based on multimodal interference in singlemode-periodically tapered small core fibre-singlemode fibre structure (SPTS) capable of improving the sensitivity. The small core fibre used in the experiments was a commercial fibre (Nufern S405 HP) with a core diameter of circa $2.5 \mu \mathrm{m}$ and a cut-off wavelength of $365 \mathrm{~nm}$. The schematic configuration of the SSCS used as the starting point to fabricate the SPTS used in the experiments is shown in Fig. 1. Light propagation in the SSCS fibre section has been simulated and analyzed in Ref. 5. In the experimental verification of Ref. 5 it was found that the power of the core mode guided in such an SSCS structure is low ( - $25 \mathrm{~dB})$ and can thus be ignored in the theoretical model, improving the agreement between the modeled and experimental results; this supports the fact that only the multimode interference between the cladding modes is essential and should be considered.

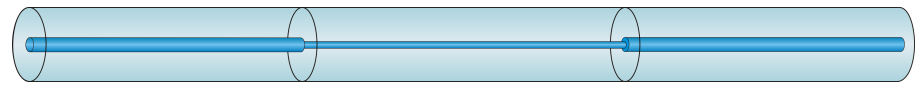

Fig. 1. Schematics of the SMF28-small core fibre-SMF28 fibre structure

Ref. 6 has shown that if the multimode section is tapered in a conventional singlemode-multimode-singlemode (SMS) structure, then strong mode interference occurs within the tapered MMF section due to the focusing effects of the left tapered transition region. Within the tapered MMF section, the excited modes of LP0m in MMF core will be partly coupled to the high-order cladding modes at the beginning of the fibre taper region and this increases the fraction of power in the evanescent field within the region of MMF cladding. This phenomenon offers a possibility to increase the intensity of multimode interference of the cladding modes in an SSCS using a tapered structure. Furthermore, by using several concatenated tapers, a periodic taper structure is created as shown in Fig. 2, consisting of an input SMF, a periodically tapered small core fibre section and an output SMF. Such an SPTS structure offers the potential to achieve a higher sensitivity than a single taper alone due to the increased cladding mode interference induced by the multiple focusing effects of the left tapered transition region.

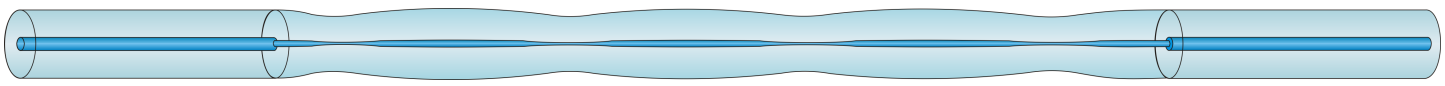

Fig. 2. SMF28-small core fibre with periodical tapers-SMF28 fibre.

\section{FABRICATION OF SPTS FIBRE STRUCTURE}

The SSCS fibre sample used as the starting point for the SPTS was manufactured from a 21 mm length of Nufern S405 HP step index SMF which was stripped, cleaved and then spliced between two standard SMF28 fibres. As shown in Fig. 3, a $\mathrm{CO}_{2}$ laser (SYNRAD, Model: 48-2KWL, with a maximum power 30 Watts at a wavelength of $10.6 \mu \mathrm{m}$ ) was employed to fabricate the tapered fibre. A ZnSe cylindrical lens with a focal length of $254 \pm 0.5 \% \mathrm{~mm}$ focused the $\mathrm{CO}_{2}$ laser beam to $\sim 200 \mu \mathrm{m}$. Beam movement was achieved using gold-coated mirrors on a motorized translation stage. A 
Labview program controlled the shutter opening and therefore the laser exposure time. One 3D translation stage was used to adjust the heating position within the small core fibre section, while a weight ( $\sim 3.5 \mathrm{~g})$ was used to apply a constant tension to the SMF end of the SSCS structure. The small core fibre was exposed to the $\mathrm{CO}_{2}$ laser beam with an output power of $10.5 \mathrm{~W}$ for $120 \mathrm{~s}$ and tapering occurred because of the tension applied to the end of the SMF. Then the laser beam was moved by a distance of $400 \mu \mathrm{m}$ along the fibre axis to irradiate and taper another segment of the fibre. Such tapering process was repeated 20 times. Consequently, periodic tapers were created on the small core fibre, as shown in Fig. 4a. Although the moving distance of the laser beam is $400 \mu \mathrm{m}$ along the fibre axis, the actual period of the tapers is circa $500 \mu \mathrm{m}$ due to the elongation of the fibre induced by tapering. The detail of one of the resulting tapers is shown in Fig. 4b, the waist diameter D is circa $90 \mu \mathrm{m}$. The transmission spectra of the SPTS fibre structure were monitored and recorded during fabrication using a Supercontinuum source (Fianium Ltd, U.K.), which delivered $50 \mathrm{~nJ}$ pulses over a broad range wavelength light $(450-1800 \mathrm{~nm})$, and a high resolution (20 pm) optical spectrum analyzer (YOKOGAWA AQ6370).

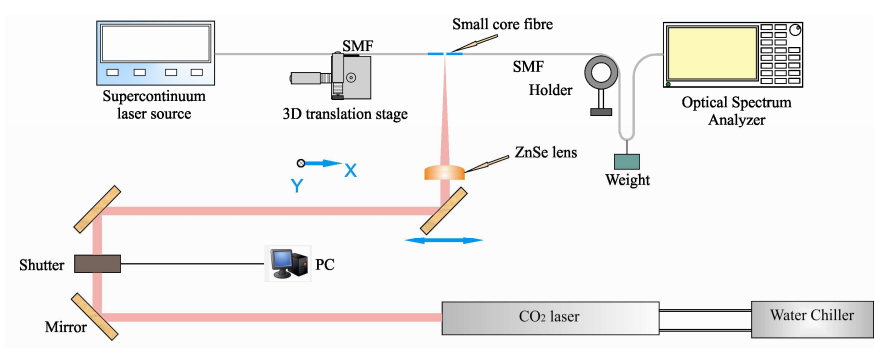

Fig. 3. Schematic of the fabrication and experimental setup.

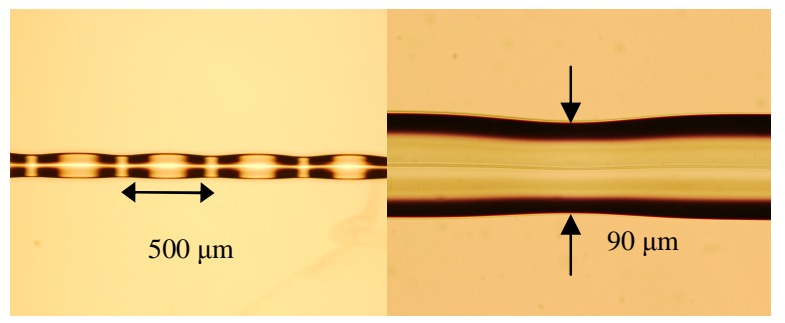

Fig. 4. Images of the small core fibre with periodic microtapers. The image was obtained by the use of a microscope (Nikon Eclipse LV100) with a $5 \times$ (left) and $20 \times$ (right) objective, respectively.

\section{RESPONSE OF SPTS TO REFRACTIVE INDEX}

An investigation of the refractive index sensing capability was performed at room temperature $\left(\sim 25^{\circ} \mathrm{C}\right)$ with a series of RI liquids (1.33 1.38 with an interval of 0.005 , RI error \pm 0.0002$)$. The RI liquids were using a dropper placed so as to cover the entire length of the SPTS fibre. Light from a supercontinuum source was launched into the SPTS and the transmitted light was collected by the optical spectrum analyzer. The measurements were carried out in the range of circa 1520-1535 $\mathrm{nm}$ as this corresponded to the location of the largest transmission dips in the transmission spectrum. The dip wavelength shifts in the wavelength range are plotted in Fig. 5 as a function of RI. As expected from the brief analysis mentioned in Sec. 1, the curve follows an essentially exponential distribution with an average sensitivity of 
$226.6 \mathrm{~nm} / \mathrm{RIU}$ over an RI range of 1.33 1.38. A maximum sensitivity of $383 \mathrm{~nm} / \mathrm{RIU}$ is achieved for RI 1.38, resulting in a resolvable index change of $4.41 \times 10^{-5}$ for a resolvable wavelength change of $0.01 \mathrm{~nm}$, which is considerably better than the experimental results presented in Ref. 5.
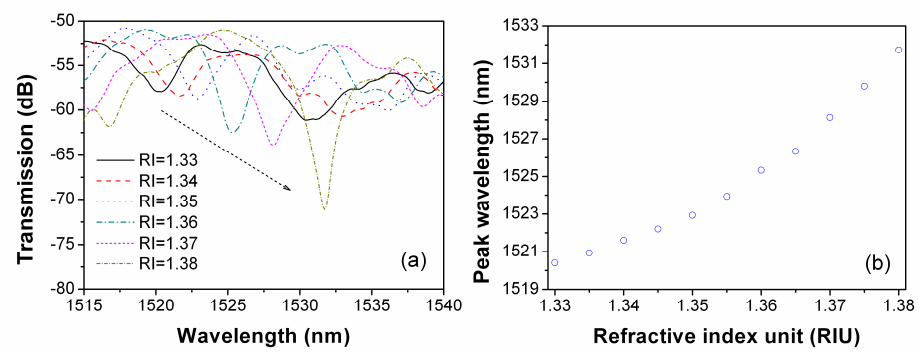

Fig. 5. (a) Measured spectral response at different surrounding RIs; (b) measured wavelength shift versus different surrounding RIs.

\section{CONCLUSION}

In conclusion, an all-fibre refractive index sensor with a range of periodical tapers configuration is proposed and investigated experimentally. A minimum sensitivity of $125 \mathrm{~nm} / \mathrm{RIU}$ with a refractive index of 1.33 and a maximum sensitivity of $383 \mathrm{~nm} / \mathrm{RIU}$ with a refractive index of 1.38 have been achieved experimentally with a $\sim 90 \mu \mathrm{m}$ periodically tapered waist diameter. Further optimization of the fibre sensor geometry will result in a more compact refractometric sensor device with improved performance.

\section{ACKNOWLEDGEMENT}

P. Wang is funded by the Irish Research Council for Science, Engineering and Technology, co-funded by the Marie-Curie Actions under FP7, G. Brambilla gratefully acknowledges the Royal Society (London) for his research fellowship, Q. Wu gratefully acknowledges the support of Science Foundation Ireland under grant no. 07 /SK /I1200.

\section{REFERENCE}

[1] Han, M., Guo F. W., and Lu, Y. F., "Optical fiber refractometer based on cladding-mode Bragg grating," Opt. Lett., 35(3), 399-401 (2010).

[2] Rindorf L., and Bang, O., "Highly sensitive refractometer with a photonic-crystal-fiber long-period grating," Opt. Lett., 33(b), 563-565 (2008).

[3] Xu F., and Brambilla, G., "Demonstration of a refractometric sensor based on optical microfiber resonator," Applied Physics Letters, 92, 101126 (2008).

[4] Wang, Q., and Farrell, G., “All-fiber multimode-interference-based refractometer sensor: proposal and design," Opt. Lett., 31(3), 317-319 (2006).

[5] Wu, Q., Semenova, Y., Wang, P., and Farrell, G., "A comprehensive analysis verified by experiment of a refractometer based on an SMF28- Small-Core Singlemode fiber (SCSMF) -SMF28 fiber structure", Journal of Optics, 13, 125401 (2011).

[6] Wang, P., Brambilla, G., Ding, M., Semenova, Y., Wu, Q., and Farrell, G., "A high sensitivity, evanescent field refractometric sensor based on tapered multimode fiber interference," Optics Letters, 36(12), 2233-2235 (2011). 